\title{
Proteomic analysis of steroid-triggered autophagic programmed cell death during Drosophila development
}

\author{
DN Martin ${ }^{1,5}$, B Balgley ${ }^{2,5}$, S Dutta ${ }^{1,5}$, J Chen ${ }^{3,5}$, P Rudnick ${ }^{2}$, J Cranford ${ }^{1}$, S Kantartzis ${ }^{1}$, DL DeVoe ${ }^{2,4}$, C Lee $e^{2,3}$ and EH Baehrecke ${ }^{\star, 1}$
}

Two morphological forms of programmed cell death, apoptosis and autophagic cell death, remove unneeded or damaged cells during animal development. Although the mechanisms that regulate apoptosis are well studied, little is known about autophagic cell death. A shotgun proteome analysis of purified dying larval salivary glands in Drosophila was used to identify proteins that are expressed during autophagic programmed cell death. A total of 5661 proteins were identified from stages before and after the onset of cell death. Analyses of these data enabled us to identify proteins from a number of interesting categories including regulators of transcription, the apoptosis, autophagy, lysosomal, and ubiquitin proteasome degradation pathways, and proteins involved in growth control. Several of the identified proteins, including the serine/threonine kinase warts (Wts), were not detected using whole-genome DNA microarrays, providing support for the importance of such high-throughput proteomic technology. Wts regulates cell-cycle arrest and apoptosis, and significantly, mutations in wts prevent destruction of salivary glands.

Cell Death and Differentiation (2007) 14, 916-923. doi:10.1038/sj.cdd.4402098; published online 26 January 2007

Programmed cell death is a fundamental component of animal development, and disruption of normal cell death is associated with several disorders including cancer and autoimmunity. ${ }^{1}$ Apoptosis and autophagic cell death are two prominent morphological forms of cell killing that occur during animal development. ${ }^{1-3}$ Apoptosis occurs when isolated cells die, and these cells are engulfed and degraded by phagocytes. ${ }^{4}$ By contrast, autophagic cell death is observed when groups of cells or entire tissues are destroyed, and these cells appear to degrade themselves with little or no help from phagocytes. ${ }^{3}$ Studies of apoptosis have resulted in the identification of a conserved cell death pathway involving caspase proteases. ${ }^{5}$ Although recent studies of cells dying by autophagic cell death have implicated some of the conserved core apoptosis genes in this morphologically distinct form of regulated cell death, ${ }^{6-8}$ the mechanisms that regulate autophagic cell death remain poorly understood.

Autophagic cell death is defined by the presence of autophagic vacuoles (also known as autophagosomes) that are used to sequester and target components of the cytoplasm for degradation by the lysosome. ${ }^{9,10}$ Autophagic vacuoles have been observed in many types of dying cells in diverse organisms, but little is known about the function of autophagy in cell death. Recent studies of mammalian cells indicate that autophagy and Atg genes are important for caspase-independent death of cells, ${ }^{11,12}$ but the relationship between autophagy and the death of cells is not clear. ${ }^{10}$ Although such studies of cell lines enable useful insights into the biochemical mechanisms that regulate cell death, it is important to study dying cells in a physiological context in vivo.

Autophagic cell death has been most frequently observed in cells dying in the context of animal development. ${ }^{1,2}$ The steroid hormone 20-hydroxyecdysone (ecdysone) triggers programmed autophagic cell death during Drosophila development. The ecdysone titer rises $10-12 \mathrm{~h}$ after puparium formation, and triggers the synchronous death of larval salivary gland cells by activating a transcription regulatory hierarchy. The ecdysone receptor, which is encoded by the nuclear receptors EcR and Usp, ${ }^{13}$ and the nuclear receptor competence factor $\beta F T Z-F 1$, activate transcription of the early genes $B R-C, E 74 A$, and $E 93^{14,15}$ These early genes are required for salivary gland cell death, and regulate transcription of the apoptosis genes rpr, hid, the Apaf-1 homolog ark, and the caspases dronc and drice. ${ }^{16-18}$ Salivary gland cell death is preceded by markers of apoptosis including DNA fragmentation and nuclear acridine orange staining. ${ }^{16}$ Although ecdysone triggers programmed cell death in Drosophila by activating the transcription of genes that encode components of the core apoptosis machinery, several differences exist in these dying cells. Salivary gland cells possess autophagic vacuoles that contain organelles including mitochondria, and do not appear to be associated with phagocytes during degradation, indicating that they die by autophagic cell death. ${ }^{6,7}$ In addition, salivary gland degradation is not completely prevented by either inhibition of caspases by $\mathrm{p} 35^{6,7}$ or loss-of-function mutations in ark and

\footnotetext{
${ }^{1}$ Center for Biosystems Research, University of Maryland Biotechnology Institute, College Park, MD, USA; ${ }^{2}$ Calibrant Biosystems, Rockville, MD, USA; ${ }^{3}$ Department of Chemistry and Biochemistry, University of Maryland, College Park, MD, USA and ${ }^{4}$ Department of Mechanical Engineering, University of Maryland, College Park, MD, USA

${ }^{*}$ Corresponding author: EH Baehrecke, Center for Biosystems Research, University of Maryland Biotechnology Institute, College Park, MD 20742-4450, USA.

Tel: 301405 7525; Fax: 301314 9075; E-mail: baehreck@umbi.umd.edu

${ }^{5}$ These authors contributed equally to this work

Keywords: Drosophila; autophagy; ecdysone; steroid; proteome

Abbreviations: (DInR), Drosophila Insulin receptor; (Kel), Kelch; (Crb), Crumbs; (Cact) Cactusl(Qua), Quail; (Hiw), Highwire; (MS), mass spectrometer

Received 21.11.06; revised 12.12.06; accepted 12.12.06; Edited by S Kumar; published online 26.1.07
} 
drice, ${ }^{19,20}$ suggesting that caspase-independent degradation mechanisms may contribute to this alternative form of cell death.

Given the importance of cell death during development, and the lack of information about autophagic cell death, we have used high-throughput genomic approaches to investigate dying salivary glands in Drosophila. Here we use a novel proteomics approach to identify proteins that are expressed before and during steroid-triggered autophagic cell death during development. Proteins that are known to function in dying salivary glands, including ecdysone-response proteins, transcription factors, caspases and caspase regulators, are expressed in dying salivary glands. In addition, new proteins that are involved in caspase-independent degradation mechanisms and growth control were also expressed in these dying cells. Significantly, the demonstration that wts functions in destruction of salivary glands illustrates the importance of complementary genomics approaches, as several proteins that were identified using proteomics, including Wts, were not detected using whole-genome DNA microarray and serial analysis of gene expression (SAGE) technologies to study these dying cells. ${ }^{18,21}$

\section{Results}

Identification of proteins expressed during autophagic cell death of salivary glands. A rise in the steroid hormone 20-hydroxyecdysone (ecdysone) triggers larval salivary gland autophagic programmed cell death. ${ }^{9,22}$ Although proteins associated with apoptosis, including caspases, are induced and participate in the destruction of salivary glands, the inhibition of caspases does not completely prevent changes associated with dying cells. ${ }^{6,19,20}$ To identify proteins that are expressed in salivary glands, soluble proteins were extracted from salivary glands dissected from wild-type animals that were staged 6 and $13 \mathrm{~h}$ after puparium formation; stages before and immediately following the rise in ecdysone that triggers cell death. Two independent samples of Trypsin-digested proteins from each stage were separated by capillary isoelectric focusing (IEF), and 12 different fractions were separated further by capillary reverse-phase liquid chromatography. These fractions were analyzed using a ThermoFinigan (Waltham, MA, USA) LCQ ion trap mass spectrometer (MS). Three MS/MS scans were performed and raw data files were processed and submitted for database searching using MASCOT (Matrix Science, Boston, MA, USA). We identified 3352 proteins in the $6 \mathrm{~h}$ samples with greater than $95 \%$ confidence, and 1111 of these proteins contained two or more distinct peptide sequences (Table S1). Similarly, 4523 proteins were identified in the $13 \mathrm{~h}$ sample with greater than $95 \%$ confidence, and 1907 of these proteins were identified based on two or more distinct peptide sequences. The total number of proteins identified in both samples is 5661 with 2214 proteins common to both 6 - and $13 \mathrm{~h}$ stages.

Proteins that are known to be involved in salivary gland programmed cell death were identified using this proteomic approach. The ecdysone regulated proteins Eip74EF (E74), Eip93F (E93), and the nuclear hormone receptors Usp and
E75B were all detected using this approach (Table S1), and this is consistent with DNA microarray studies ${ }^{18}$ and previous studies of these transcription regulators. ${ }^{17,23-25}$ Caspase proteases function in salivary gland cell death, $, 7,16,26$ and several proteins that function during apoptosis were detected including the caspase activator Ark, and the caspases Strica/Dream and Nc/Dronc, consistent with DNA microarray studies. ${ }^{18}$ These data provide confidence that proteins that are known to be involved in salivary gland programmed cell death can be identified using this shotgun proteomics approach, and that this can provide a valuable overview of the proteome in dying cells.

Identification of new salivary gland proteins. Although the identification of several known salivary gland proteins provided evidence that this novel proteome technology is sensitive, the validation of the presence of newly discovered proteins is critical to endear confidence before investing effort in reverse genetic studies. We analyzed our data for previously studied proteins so that we could use available antibodies to validate our salivary gland proteome data using immunohistochemical staining. Salivary glands were isolated from wild-type Canton $S$ animals staged to 6 and $13 \mathrm{~h}$ after puparium formation, fixed and stained with available antibodies against the Drosophila insulin receptor (DInR), Kelch (Kel), Crumbs (Crb), Cactus (Cact), Quail (Qua), Highwire (Hiw), and Osa. ${ }^{27-33}$ We had previously shown that nuclear Lamin DmO and $\alpha$-spectrin are expressed in dying salivary glands, ${ }^{7}$ and these antibodies were used as positive controls.

Whole-genome DNA microarray studies indicated that several of the identified salivary gland proteins are transcribed in these cells, ${ }^{18}$ and we intitiated our validation by evaluating the presence of these proteins (Figure 1). DInR was identified based on the detection of multiple distinct peptides and the presence of RNA using DNA microarrays, and DInR antigen is present in salivary gland cells. The actin-binding protein Kel and the cell polarity protein Crb were both detected using antibodies as expected based on the identification of multiple distinct peptides from each of these proteins, as well as detection of their RNAs in previous microarray studies. We only detected a single peptide from the IkB protein Cact, and the presence of this nuclear factor kappa $B$ regulator was validated using antibodies as expected based on the presence of Cact RNA in microarray studies. We then addressed if the actin binding protein Qua was present in these cells, as quail RNA was present at low levels in DNA microarray studies, ${ }^{18}$ and we only detected a single Qua peptide in our proteomic studies. The presence of Qua antigen in salivary glands provided greater confidence that the detection of single peptides using this approach can lead to the identification of proteins.

The identification of low abundance proteins, and proteins that may not be regulated at the level of RNA transcription, provides one of the greatest incentives for the development of sensitive high-throughput proteomics technologies. To test if we could detect proteins that were absent in our wholegenome microarray studies, ${ }^{18}$ but were present in our proteomics data, we stained salivary glands with antibodies against Hiw and Osa (Figure 1). Hiw encodes a putative 


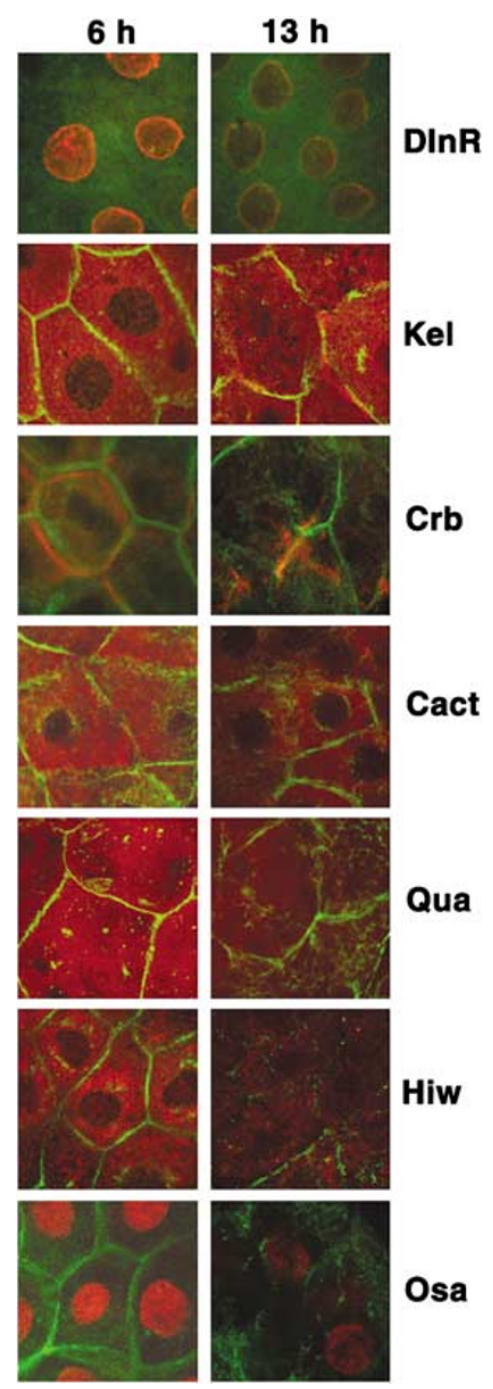

Figure 1 Verification of proteome results by immunohistochemistry. Salivary glands of staged wild-type Canton $S$ animals were stained with antibodies against either DInR (green) and nuclear Lamin DmO (red), or $\alpha$ - Spectrin (green) and experimental antibodies (in red) against Kel, Crb, Cact, Qua, Hiw, Osa

Ubiquitin ligase that regulates synaptic growth, ${ }^{34}$ and the identification of multiple distinct peptides was validated based on Hiw localization in the cytoplasm of salivary gland cells. Similar to Hiw, the transcription regulator Osa was not detected in our previous DNA microarray studies, but Osa protein was present in the nuclei of salivary gland cells as expected based on the identification of multiple Osa peptides. Although our previous use of this proteomics approach to identify yeast proteins indicated the robust nature of this technology, ${ }^{35}$ these data provide further evidence of its strength by identification of numerous proteins from a complex animal with a larger genome.

We identified many interesting proteins using this novel high-throughput technology (Table S1), thus our next interest was to investigate what signaling pathways and cell processes may be involved in the autophagic cell death of salivary glands. Transcription is known to play an important role in this steroid-triggered cell death, and the identification of several new proteins in this category including DHR96, Cactus, Bunched, Hsf and the Mediator/Trap proteins MED1, MED14, and MED17 (Table 1) reflect the importance of the regulation of RNA levels in these dying cells. Little is known about the transcription coactivators and corepressors that are involved in programmed cell death, and the identification of several proteins in these categories, including Hcf, Nej, Tai, Brm, Gug, H, Hira, and Smarter (Smr), provides a starting point for investigating their function in autophagic programmed cell death (Table 1).

Salivary glands rapidly degrade during autophagic programmed cell death, ${ }^{6}$ and we hypothesize that many categories of proteins may contribute to this process. Supporting this idea, we identified proteins involved in apoptosis, the ubiquitin proteasome system, autophagy, lysosome biogenesis, and noncaspase proteases (Table 2, Table S2). Several proteins that function during apoptosis were detected including the Bcl-2 family members Debcl and Buffy, the ubiquitin ligase Morgue, the caspase activator Ark, and the caspases Dream/Strica and Nc/Dronc. The ubiquitin proteasome system serves an important role in the degradation of short-lived proteins including caspase regulators. In addition to the identification of several proteasome components (Table S2), numerous proteins that are involved in the regulation of ubiquitination were detected including Bruce and Ago, as well as many others (Table 2). Autophagic vacuoles form following the initiation of salivary gland cell death. Although the mechanism(s) by which autophagy is regulated is not fully understood in these cells, the presence of the autophagy proteins Atg1, Atg2, Atg4, Atg6, Atg9, and the Atg18-like protein CG8678 are consistent with this catabolic process being involved in salivary gland cell death (Table 2). Autophagy requires lysosomes for the degradation of cargo, and it is intriguing that several proteins involved in lysosome biogenesis and organization, such as orange, ruby, and garnet that were originally isolated based on defects in eye pigmentation, were identified (Table 2). Given this result, we tested if lysosomes are more abundant in later stages of salivary gland cell death by using a fusion between the lysosomal associated membrane protein 1 (LAMP1) and horse radish peroxidase (HRP) that has been previously used to detect lysosomes in Drosophila. ${ }^{36}$. Salivary glands isolated from animals $12 \mathrm{~h}$ after puparium formation have large vacuoles in every cell and little LAMP1-HRP staining (Figure 2). By contrast, salivary glands dissected $14 \mathrm{~h}$ after puparium formation possess increased LAMP1-HRP staining that is localized to perinuclear lysosome membranes (Figure 2). These results are consistent with the timing of the induction of autophagy, and Atg RNA and proteins, that immediately precede cell degradation ${ }^{6,18}$ (Table 2), and suggest that lysosome biogenesis may be important for completion of salivary gland degradation.

Dying salivary glands exhibit dynamic changes in structure including the loss and formation of different types of vacuoles ${ }^{6}$ (Figure 2), and the actin cytoskeleton is reorganized independent of caspase activation. ${ }^{7}$ Although some of these changes could be caused by the cleavage of proteins by proteases, other mechanisms may regulate reorganization of the cytoskeleton and movement of structures during autophagic cell death. Consistent with this hypothesis, we 
Table 1 Summary of new transcription regulators that were identified in dying salivary glands

\begin{tabular}{|c|c|c|c|c|c|c|c|}
\hline \multirow[b]{2}{*}{ Category } & \multirow[b]{2}{*}{ Name } & \multirow[b]{2}{*}{ FBgn\# } & \multirow[b]{2}{*}{ Notes } & \multicolumn{2}{|c|}{$6 \mathrm{~h}$} & \multicolumn{2}{|c|}{$13 \mathrm{~h}$} \\
\hline & & & & dpep & tpep & dpep & tpep \\
\hline \multicolumn{8}{|c|}{ Transcription Regulators } \\
\hline & DHR96* & FBgn0015240 & Nuclear receptor & 0 & 0 & 6 & 10 \\
\hline & Cact $^{*}$ & FBgn0000250 & IkB, NFkB regulator & 0 & 0 & 1 & 1 \\
\hline & Bun* & FBgn0010460 & & 1 & 1 & 1 & 1 \\
\hline & $\mathrm{Hsf}^{\star}$ & FBgn0001222 & Heat shock factor & 0 & 0 & 1 & 1 \\
\hline & MED1* & FBgn0037109 & Mediator/Trap220 & 3 & 3 & 4 & 6 \\
\hline & MED14* & FBgn0035145 & Mediator/Trap170 & 2 & 2 & 3 & 5 \\
\hline & MED17* & FBgn0038578 & Mediator/Trap80 & 0 & 0 & 2 & 2 \\
\hline \multicolumn{8}{|c|}{ Transcription Coactivators } \\
\hline & $\operatorname{Hcf}^{*}$ & FBgn0039904 & Heat cell factor & 3 & 3 & 4 & 6 \\
\hline & $\mathrm{Nej}^{*}$ & FBgn0015624 & Nejire & 4 & 10 & 3 & 14 \\
\hline & Tai & FBgn0041092 & Taiman & 4 & 19 & 5 & 15 \\
\hline & $\mathrm{Brm}^{*}$ & FBgn0000212 & Brahma & 1 & 1 & 2 & 2 \\
\hline \multicolumn{8}{|c|}{ Transcription Corepressors } \\
\hline & Gug & FBgn0010825 & Grunge & 4 & 4 & 2 & 2 \\
\hline & $\mathrm{H}^{*}$ & FBgn0001169 & Hairless & 0 & 0 & 3 & 3 \\
\hline & Hira & FBgn0022786 & & 0 & 0 & 1 & 1 \\
\hline & Smr* & FBgn0024308 & Smarter & 3 & 3 & 5 & 5 \\
\hline
\end{tabular}

Categories are based on association with biological or biochemical properties. FBgn numbers and names of proteins are based on Flybase annotation (http:// flybase.bio.indiana.edu/). The number of distinct peptides (dpep) and total peptides (tpep) identified in samples from each stage of salivary gland extract are indicated. Proteins that are predicted to be present based on previous whole-genome DNA microarray studies ${ }^{18}$ are indicated with an asterisk

Table 2 Summary of new proteins that function in degradation that were identified in dying salivary glands

\begin{tabular}{|c|c|c|c|c|c|c|c|}
\hline \multirow[b]{2}{*}{ Category } & \multirow[b]{2}{*}{ Name } & \multirow[b]{2}{*}{ FBgn\# } & \multirow[b]{2}{*}{ Notes } & \multicolumn{2}{|c|}{$6 \mathrm{~h}$} & \multicolumn{2}{|c|}{$13 \mathrm{~h}$} \\
\hline & & & & dpep & tpep & dpep & tpep \\
\hline \multicolumn{8}{|l|}{ Apoptosis } \\
\hline & Debcl & FBgn0029131 & Bcl-2 family & 0 & 0 & 1 & 1 \\
\hline & Buffy & FBgn0040491 & Bcl-2 family & 1 & 1 & 1 & 1 \\
\hline & Morgue & FBgn0027609 & Ubiquitin ligase & 0 & 0 & 2 & 3 \\
\hline & Ark $^{*}$ & FBgn0024252 & Apaf-1 & 0 & 0 & 2 & 2 \\
\hline & Dream* & FBgn0033051 & Strica, Caspase & 0 & 0 & 1 & 1 \\
\hline & $\mathrm{Nc}^{*}$ & FBgn0026404 & Dronc, Caspase & 0 & 0 & 1 & 1 \\
\hline \multicolumn{8}{|l|}{ Autophagy } \\
\hline & Atg1 & FBgn0037679 & & 0 & 0 & 1 & 1 \\
\hline & Atg2* & FBgn0035373 & & 1 & 1 & 5 & 6 \\
\hline & Atg $4^{*}$ & FBgn0038325 & & 0 & 0 & 3 & 4 \\
\hline & Atg6* & FBgn0039144 & Beclin1 & 0 & 0 & 2 & 2 \\
\hline & Atg $^{*}$ & FBgn0034110 & & 1 & 1 & 3 & 5 \\
\hline & CG8678* & FBgn0032935 & Atg-18 like & 0 & 0 & 1 & 1 \\
\hline \multicolumn{8}{|c|}{ Ubiquitin Conjugation } \\
\hline & Bruce $^{*}$ & FBgn0037808 & Ubiquitin ligase & 4 & 5 & 4 & 5 \\
\hline & Ago & FBgn0041171 & Ubiquitin ligase & 0 & 0 & 3 & 3 \\
\hline & Cul- $4^{*}$ & FBgn0033260 & Ubiquitin ligase & 2 & 2 & 1 & 1 \\
\hline & HERC2* & FBgn0031107 & Ubiquitin ligase & 3 & 3 & 12 & 17 \\
\hline & $\mathrm{Hyd}^{*}$ & FBgn0002431 & Ubiquitin ligase & 6 & 6 & 8 & 11 \\
\hline & Lack & FBgn0029006 & Ubiquitin ligase & 0 & 0 & 4 & 4 \\
\hline & Ubp64E* & FBgn0016756 & Ubiquitin protease & 2 & 2 & 2 & 2 \\
\hline \multicolumn{8}{|c|}{ Lysosome Biogenesis } \\
\hline & Or & FBgn0003008 & Orange & 1 & 1 & 0 & 0 \\
\hline & $\mathrm{Rb}$ & FBgn0003210 & Ruby & 1 & 1 & 1 & 1 \\
\hline & G & FBgn0001087 & Garnet & 0 & 0 & 2 & 3 \\
\hline & Dip-B & FBgn0000454 & Leucyl aminopeptidase & 1 & 1 & 3 & 3 \\
\hline & Ect3 $^{\star}$ & FBgn0037977 & $\beta$-galactosidase activity & 0 & 0 & 1 & 1 \\
\hline & $\mathrm{NPC} 1^{*}$ & FBgn0024320 & Niemann-Pick Type C-1 & 0 & 0 & 2 & 3 \\
\hline & Bchs & FBgn0043362 & Lysosome transport & 0 & 0 & 5 & 5 \\
\hline
\end{tabular}

Categories are based on association with biological or biochemical properties. FBgn numbers and names of proteins are based on Flybase annotation (http:// flybase.bio.indiana.edu/). The number of distinct peptides (dpep) and total peptides (tpep) identified in samples from each stage of salivary gland extract are indicated. Proteins that are predicted to be present based on previous whole-genome DNA microarray studies ${ }^{18}$ are indicated with an asterisk 
identified a large number of proteins that participate in changes in cell organization including GTPases, ARFs, ARPs, formin homology proteins, and motors (Table S3).
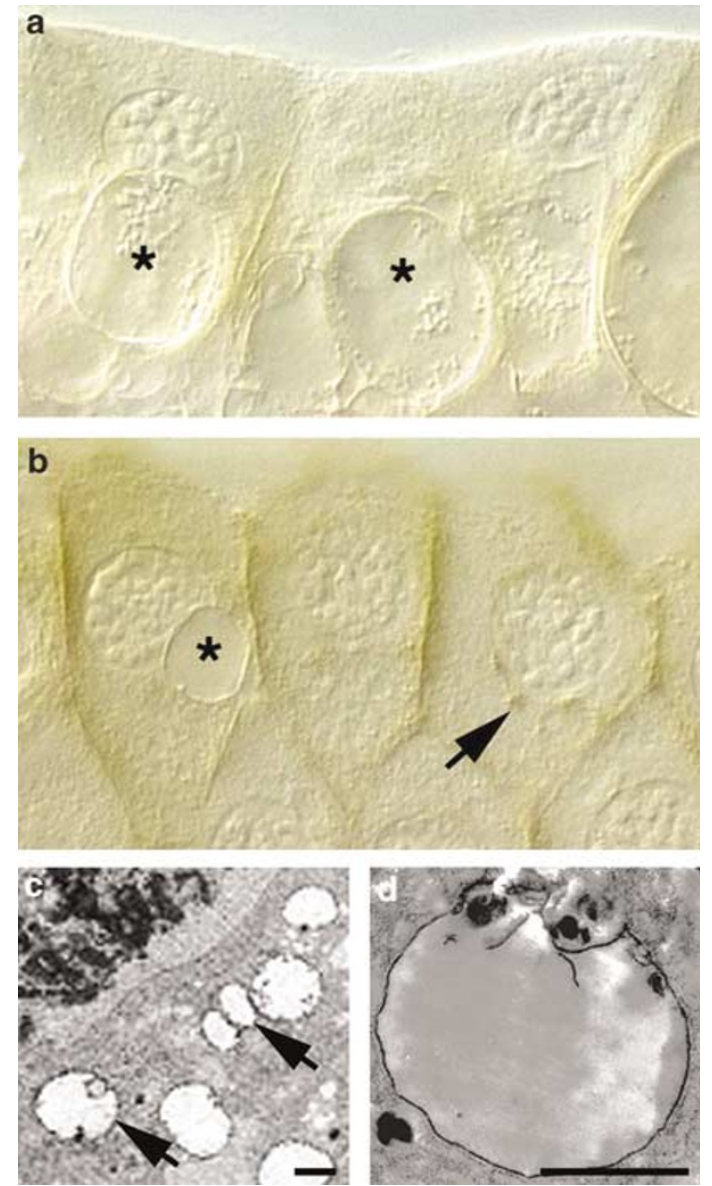

Figure 2 Lysosomes are induced just before salivary gland cell death. Salivary glands of animals that express the lysosome protein Lamp1 fused to HRP were staged $12 \mathrm{~h}(\mathbf{a})$ and $14 \mathrm{~h}(\mathrm{~b}-\mathrm{d})$ after puparium formation to visualize lysosomes by either light ( $\mathbf{a}$ and $\mathbf{b}$ ) or transmission electron microscopy (c and $\mathbf{d})$. (a) Twelve-hour salivary glands have large vacuoles in every cell (asterisks) and little HRP staining. (b-d) Salivary glands dissected $14 \mathrm{~h}$ after puparium formation have either few or no large vacuoles (asterisks), and these cells possess increased HRP staining that is localized to perinuclear lysosome membranes (arrows). Scale bars in c and d, $1 \mu \mathrm{m}$
An intricate relationship exists between cell growth and proliferation, and programmed cell death during animal development. Growth and proliferation arrest are often associated with the activation of apoptosis, but the relationship between growth and autophagic cell death is unclear. The insulin receptor ( $\mathrm{InR}$ ) signaling pathway is an important mechanism for the regulation of growth. InR signaling may be relevant to autophagic cell death, as autophagy is influenced by the activity of several proteins within this pathway. ${ }^{37} \mathrm{We}$ identified many components of this pathway including $\operatorname{lnR}$, the class I phosphotidylinositol 3 kinase (PI3K) PI3K92E, the tuberous sclerosis protein TSC1, the target of rapamycin (Tor), as well as 2 other InR-like proteins (Table 3). In addition to our identification of the class I PI3K, we also identified a protein encoding the class II PI3K68D (Table 3).

Wts functions in salivary gland cell death. Mutations in the serine/threonine kinase warts (Wts) result in overgrowth of adult tissues, ${ }^{38}$ and it was of interest that we identified two distinct Wts peptides in $13 \mathrm{~h}$ salivary glands (Table S1). Wts is among the novel proteins identified in this study that were not identified using DNA microarrays. ${ }^{18}$ As Wts has been previously linked to the regulation of cell-cycle arrest and apoptosis, and encodes a tumor suppressor, we tested if wts function is required for autophagic cell death of salivary glands.

Strong loss-of-function wts mutants are lethal before puparium formation. Therefore, we analyzed the weak wts ${ }^{P 2}$ allele for defects in salivary gland cell death. ${ }^{38}$ Homozygous $w t s^{P 2}$ and $w t s^{P 2}$ /wild-type control pupae were aged $12 \mathrm{~h}$ after future adult head eversion which is $8 \mathrm{~h}$ after larval salivary gland cell death is complete in wild-type flies. ${ }^{6}$ These pupae were embedded in paraffin, sectioned, and stained for examination of cell death defects. In sections of control wts ${ }^{P 2}$ Wild-type, salivary glands are destroyed (Figure 3). By contrast, $100 \%$ of homozygous wts pupae had defects in salivary gland destruction (Figure 3 ), with $32 \%$ having intact salivary glands and $68 \%$ having fragmented salivary glands that are not properly degraded. Although homozygous wts pupae often exhibited over growth of imaginal tissues, this did not prevent either head eversion or the normal development of other structures. As Wts functions in a pathway with Hippo (Hpo), we also tested if expression of a previously studied

Table 3 Summary of growth regulators that were identified in dying salivary glands

\begin{tabular}{|c|c|c|c|c|c|c|c|}
\hline \multirow[b]{2}{*}{ Category } & \multirow[b]{2}{*}{ Name } & \multirow[b]{2}{*}{ FBgn\# } & \multirow[b]{2}{*}{ Notes } & \multicolumn{2}{|c|}{$6 \mathrm{~h}$} & \multicolumn{2}{|c|}{$13 h$} \\
\hline & & & & dpep & tpep & dpep & tpep \\
\hline \multicolumn{8}{|l|}{ Growth } \\
\hline & PI3K68D* & FBgn0015278 & Class II PI3K & 0 & 0 & 1 & 1 \\
\hline & PI3K92E* & FBgn0015279 & Class I PI3K & 2 & 3 & 1 & 1 \\
\hline & $\ln R^{*}$ & FBgn0013984 & Insulin receptor & 3 & 7 & 5 & 9 \\
\hline & CG3837* & FBgn0038279 & Insulin-like receptor & 3 & 4 & 1 & 7 \\
\hline & CG10702 & FBgn0032752 & Insulin-like receptor & 5 & 6 & 8 & 15 \\
\hline & TSC1 & FBgn0026317 & Insulin receptor signaling & 1 & 1 & 3 & 8 \\
\hline & Tor & FBgn0021796 & Target of rapamycin & 0 & 0 & 1 & 1 \\
\hline
\end{tabular}

Categories are based on association with biological or biochemical properties. FBgn numbers and names of proteins are based on Flybase annotation (http:// flybase.bio.indiana.edu/). The number of distinct peptides (dpep) and total peptides (tpep) identified in samples from each stage of salivary gland extract are indicated. Proteins that are predicted to be present based on previous whole-genome DNA microarray studies ${ }^{18}$ are indicated with an asterisk 

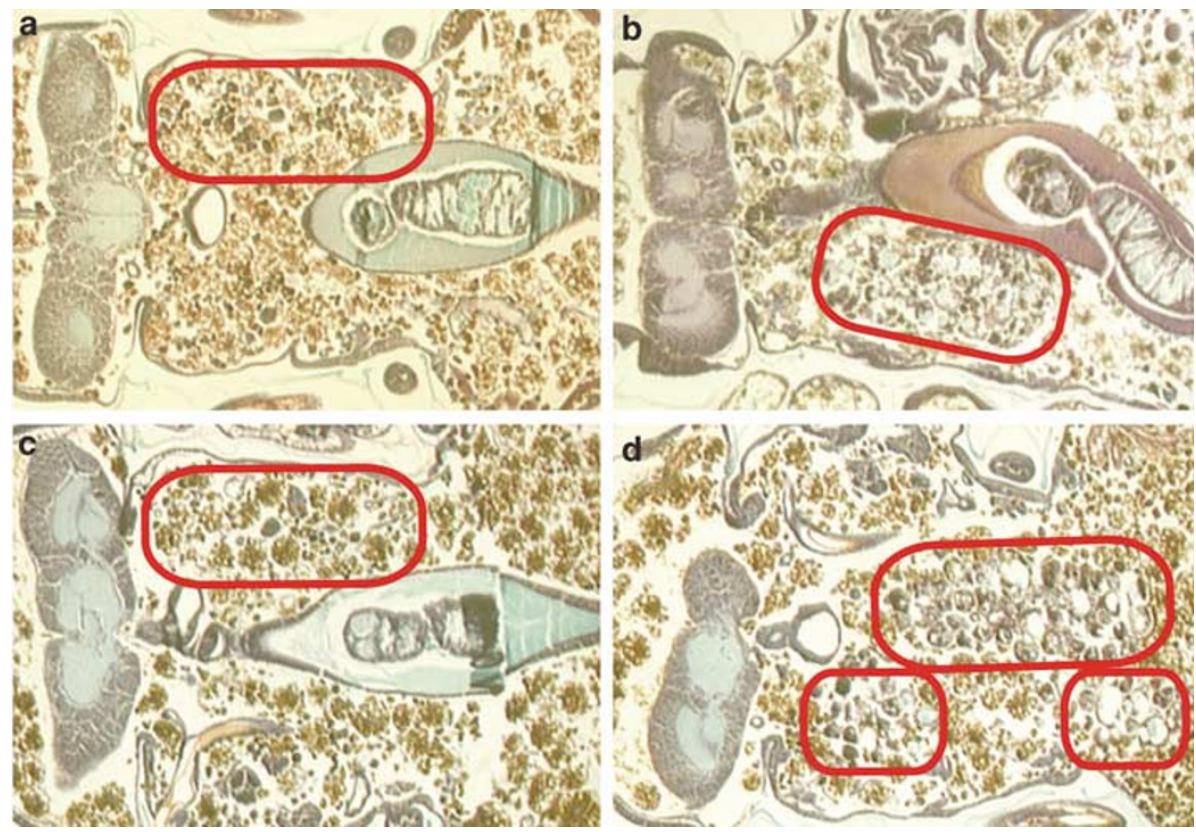

Figure 3 Wts and Hpo function in salivary gland autophagic cell death. Paraffin sections of pupae aged $12 \mathrm{~h}$ after head eversion. (a) In control wts ${ }^{P 2} /$ CantonS wild-type pupae salivary glands have degraded $(n=13)$. (b) $100 \%$ of $\mathrm{wts}^{P 2} / \mathrm{wts}^{P 2}$ mutant pupae possess salivary glands $24 \mathrm{~h}$ after puparium formation $(n=22)$. (c) Control, either UAS$h p o / R / C a n t o n S$ or $f k h-G A L 4 / C a n t o n S$, pupae lack salivary glands $(n=16)$. (d) Experimental $f k h$-GAL4 UAS-hpolR pupae possess partially degraded salivary glands $24 \mathrm{~h}$ after puparium formation in $40 \%$ of animals $(n=15)$. Red circles mark the absence of salivary glands in controls (a and $\mathbf{c}$ ), and persistent salivary glands and gland fragments in experimental wts mutant and hpolR animals (b and $\mathbf{d})$

hippoRNAi construct $(h p o l R)^{39}$ in salivary glands prevented salivary gland cell death by analysis of paraffin sections of pupae that were aged $12 \mathrm{~h}$ after future adult head eversion. Control animals, either UAS-hpolR/wild type or $f k h-G A L 4 /$ wild type, did not possess salivary glands (Figure 3). Animals that express $h p o l R$ in salivary glands had fragmented remnants of salivary glands in $40 \%$ of the specimens (Figure 3 ). These results indicate that wts and hpo function in autophagic cell death of salivary glands.

\section{Discussion}

Although much is known about the mechanisms that regulate apoptosis, little is known about alternative morphological forms of cell killing including autophagic cell death. Here we have used a high-throughput approach to identify proteins in dying salivary glands. This resulted in the identification of numerous factors that we expected based on previous wholegenome microarray and SAGE studies, ${ }^{18,21}$ but we also identified proteins that were not previously known to be expressed in dying salivary glands. These data indicate that it is important to combine proteomic approaches with genomic technologies designed to detect RNAs to get a complete overview of the possible players involved in a cellular process. The presence of a protein within a dying cell is not evidence that this factor functions in cell death, and caution should be taken before implicating innocent bystanders as being guilty by association. Therefore, such genomic approaches will be most powerful when they are combined with genetic approaches, and we now have a list of cellular processes and signaling pathways that need to be investigated by analyses of mutants.

Previous studies have shown that a rise in the steroid ecdysone triggers a transcription cascade that activates a caspase-dependent autophagic cell death of salivary glands. ${ }^{9}$ This model has strong support, because mutations in primary ecdysone-response transcription regulators prevent salivary gland cell death, ${ }^{6}$ and these mutants also possess decreased levels of caspases and caspase regulators. ${ }^{7}$ However, the inhibition of caspases and caspase mutants do not prevent all of the cellular changes associated with the death of salivary glands, $, 7,19,40$ and suggest that additional noncaspase degradation mechanisms may be involved in the physiological destruction of these cells. Consistent with this hypothesis, our genomic studies indicate that multiple noncaspase degradation systems, including autophagy, the ubiquitin proteasome, and the lysosome, may be involved in the death of salivary glands. Furthermore, the presence and requirement for Wts that is not ecdysone-regulated indicates that post-translational mechanisms also contribute to the death of salivary glands.

Analyses of wts and hpo indicate that at least some of the mechanisms that regulate tissue growth control also function during autophagic cell death, but additional studies are needed to determine the relationship between cell growth and autophagic cell death. Many growth regulators, including several proteins in the Wts and PI3K pathways, depend on phosphorylation to modulate their activities, and studies of post-translational modification of proteins in dying salivary glands should provide insights into the mechanisms that mediate their cell death. The relationship between different 
signaling pathways, degradation mechanisms, and how these factors contribute to the autophagic cell death morphology is not clear, and it is important to resolve how these mechanisms integrate to result in a physiological cell removal strategy. These studies provide an important foundation for future genetic and biochemical studies of autophagic programmed cell death by providing a comprehensive description of the proteins that are present in dying salivary glands.

\section{Materials and Methods \\ Protein extraction and proteomics. Soluble protein extracts were obtained from wild-type Canton $S$ salivary glands that were dissected from animals staged 6 and $13 \mathrm{~h}$ after puparium formation. Salivary glands were homogenized in $40 \mathrm{~mm}$ Tris buffer containing $1 \mathrm{~mm}$ phenlymethylsulfonyl fluoride, $1 \mu \mathrm{m}$ Pepstatin A, $1 \mu \mathrm{m}$ Leupeptin, and incubated for $5 \mathrm{~min}$ at $100^{\circ} \mathrm{C}$. Proteins were denatured, reduced and alkylated in $20 \mathrm{mM}$ Tris buffer containing $8 \mathrm{M}$ urea, $0.1 \mathrm{M}$ dithiothreitol (DTT) and $0.1 \mathrm{~m}$ iodoacetamide. Denatured, reduced and alkylated proteins were reconstituted in a solution of $10 \mathrm{mM}$ Tris, $0.4 \mathrm{M}$ urea and $10 \mathrm{mM}$ DTT using regenerated cellulose membrane filters (Millipore, Billerica, MA, USA) with a 5000 molecular weight cutoff. Trypsin (1:50, w/w, Promega modified sequencing grade, Madison, WI, USA) was added and the mixture was incubated at $37^{\circ} \mathrm{C}$ for overnight. \\ Independent samples of trypsin-digested protein from each sample were separated by capillary isoelectric focusing. Twelve different IEF fractions were separated further by capillary reverse phase liquid chromatography, and analyzed using a ThermoFinigan LCQ ion trap MS. Three MS/MS scans were performed on the top three ions from the preceding MS scan. These data were processed using Distiller (Matrix Science) and submitted for database analyses using MASCOT (Matrix Science). The database that was searched was a non-redundant compilation of the Swiss-plot and TREMBL Drosophila melanogaster data sets available from $\mathrm{EBI}$.}

Immunohistochemistry. Wild-type Canton S salivary glands were dissected from animals staged 6 and $13 \mathrm{~h}$ after puparium formation at $25^{\circ} \mathrm{C}$, fixed in $4 \%$ paraformaldehyde/heptane for $20 \mathrm{~min}$ at room temperature, blocked in phosphatebuffered saline containing $1 \%$ BSA and $0.1 \%$. Triton-X (PBSBT), and incubated with primary antibodies for $16 \mathrm{~h}$ at $4^{\circ} \mathrm{C}$. Salivary glands were washed for $2 \mathrm{~h}$ in PBSBT, incubated with appropriate secondary antibodies for two hours at room temperature and washed for another $2 \mathrm{~h}$ in PBSBT at room temperature. Salivary glands were mounted in Vectashield (Vector Laboratories) and examined using a Zeiss Axiovert $100 \mathrm{M}$ confocal microscope.

Histology. UAS-HRP-LAMP1 transgenic flies were kindly provided by H. Kramer. ${ }^{36}$ Salivary glands were dissected from fkh-GAL4; UAS-HRP. LAMP1animals at specific stages during cell death. Salivary glands were fixed for $10 \mathrm{~min}$ in $2.5 \%$ glutaraldehyde, rinsed in PBS, and incubated in $0.5 \mathrm{mg} / \mathrm{ml} \mathrm{DAB}$ and $0.03 \%$ hydrogen peroxide. Staining of 12 and 14 -h salivary glands in DAB was done for the same period of time so that these samples could be compared. For whole mount analyses, salivary glands were then dehydrated, mounted in methyl salicylate, and examined by light microscopy. For examination of thin sections by transmission electron microscopy, DAB-stained salivary glands were fixed for an additional hour in $2.5 \%$ glutaraldehyde, postfixed in $4 \%$ osmium tetroxide for $1 \mathrm{~h}$, embedded in Spurr's resin, sectioned, and analyzed using a Zeiss EM 10 transmission electron microscope.

For paraffin sections, control (either wts ${ }^{P 2} /$ CantonS, UAS-hpolR/CantonS, or fkh-GAL4/CantonS) and experimental (either wts ${ }^{P 2} / w^{P 2}{ }^{P}$ or fkh-GAL4; UAS-hpolR) animals were maintained at $25^{\circ} \mathrm{C}$ and aged to $12 \mathrm{~h}$ after head eversion. Whole pupae were fixed in $1 \%$ glutaraldehyde, $4 \%$ formaldehyde and $5 \%$ acetic acid in $80 \%$ ethanol at $4^{\circ} \mathrm{C}$. Fixed pupae were dehydrated through an ethanol series, cleared in xylenes and infiltrated with paraffin. Sections were cut at $7 \mu \mathrm{m}$, stained with Weigert's hematoxylin and Pollak Trichrome and examined and photographed using a Zeiss Axiophot II microscope.

Acknowledgements. We thank B Hay, D Branton, P Fisher, L Pick, $H$ Kramer, G Halder, N Tapon, and the Developmental Studies Hybridoma Bank for antibodies and fly stocks, and D Berry for helpful comments and discussions. This work was supported by NIH NRSA GM067563 to DNM, an HHMI Undergraduate Research Fellowship to SK, and NIH grants CA107988 to BMB, GM073723 to CL, and GM59136 to EHB.

1. Baehrecke EH. How death shapes life during development@ Nature Reviews Mol. Cell Biol 2002; 3: 779-787.

2. Clarke PGH. Developmental cell death: morphological diversity and multiple mechanisms. Anat Embryol 1990; 181: 195-213

3. Schweichel J-U, Merker H-J. The morphology of various types of cell death in prenatal tissues. Teratology 1973; 7: 253-266.

4. Kerr JF, Wyllie AH, Currie AR. Apoptosis: a basic biological phenomenon with wideranging implications in tissue kinetics. Br J Cancer 1972; 26: 239-257.

5. Shi Y. Mechanisms of Caspase Activation and Inhibition during Apoptosis. Mol Cell 2002 9: $459-470$.

6. Lee C-Y, Baehrecke EH. Steroid regulation of autophagic programmed cell death during development. Development 2001; 128: 1443-1455

7. Martin DN, Baehrecke EH. Caspases function in autophagic cell death in Drosophila Development 2004; 131: 275-284

8. Debnath J, Mills KR, Collins NL, Reginato MJ, Muthuswamy SK, Brugge JS. The role of apoptosis in creating and maintaining luminal space within normal and oncogeneexpressing mammary acini. Cell 2002; 111: 29-40.

9. Baehrecke EH. Autophagic programmed cell death in Drosophila. Cell Death Differ 2003 10: $940-945$

10. Baehrecke EH. Autophagy: dual roles in life and death? Nat Rev Mol Cell Biol 2005; 6 : 505-510.

11. Yu L, Alva A, Su H, Dutt $P$, Freundt E, Welsh $S$ et al. Regulation of an ATG7-beclin 1 program of autophagic cell death by caspase-8. Science 2004; 304: 1500-1502.

12. Shimizu S, Kanaseki T, Mizushima N, Mizuta T, Arakawa-Kobayashi S, Thompson CB et al. Role of Bcl-2 family proteins in a non-apoptotic programmed cell death dependent on autophagy genes. Nature Cell Biol 2004; 6: 1221-1228.

13. Yao T-P, Segraves WA, Oro AE, McKeown M, Evans RM. Drosophila ultraspiracle modulates ecdysone receptor function via heterodimer formation. Cell 1992; 71 63-72

14. Broadus J, McCabe JR, Endrizzi B, Thummel CS, Woodard CT. The Drosophila $\beta \mathrm{FTZ}-\mathrm{F} 1$ orphan nuclear receptor provides competence for stage-specific responses to the steroid hormone ecdysone. Mol Cell 1999; 3: 143-149.

15. Woodard CT, Baehrecke EH, Thummel CS. A molecular mechanism for the stagespecificity of the Drosophila prepupal genetic response to ecdysone. Cell 1994; 79 607-615

16. Jiang $\mathrm{C}$, Baehrecke $\mathrm{EH}$, Thummel CS. Steroid regulated programmed cell death during Drosophila metamorphosis. Development 1997; 124: 4673-4683.

17. Lee C-Y, Wendel DP, Reid P, Lam G, Thummel CS, Baehrecke EH. E93 directs steroidtriggered programmed cell death in Drosophila. Mol Cell 2000; 6: 433-443.

18. Lee C-Y, Clough EA, Yellon P, Teslovich TM, Stephan DA, Baehrecke EH. Genome-wide analyses of steroid- and radiation-triggered programmed cell death in Drosophila. Curr Biol 2003; 13: 350-357.

19. Mills K, Daish T, Harvey KF, Pfleger CM, Hariharan IK, Kumar S. The Drosophila melanogaster Apaf-1 homologue ARK is required for most, but not all, programmed cell death. J Cell Biol 2006; 172: 809-815.

20. Muro I, Berry DL, Huh JR, Chen CH, Huang H, Yoo SJ et al. The Drosophila caspase Ice is important for many apoptotic cell deaths and for spermatid individualization, a nonapoptotic process. Development 2006; 133: 3305-3315.

21. Gorski SM, Chittaranjan S, Pleasance ED, Freeman JD, Anderson CL, Varhol RJ et al. A SAGE Approach to discovery of genes involved in autophagic cell death. Curr Biol 2003 13: 358-363.

22. Baehrecke EH. Steroid regulation of programmed cell death during Drosophila development. Cell Death Differ 2000; 7: 1057-1062.

23. Yao T-P, Forman BM, Jiang Z, Cherbas L, Chen JD, McKeown M et al. Functional ecdysone receptor is the product of EcR and ultraspiracle genes. Nature 1993; 366 476-479.

24. Hill RJ, Segraves WA, Choi D, Underwood PA, Macavoy E. The reaction with polytene chromosomes of antibodies raised against Drosophila E75 protein. Insect Biochem Mol Biol 1993; 23: 99-104.

25. Boyd L, O'Toole E, Thummel CS. Patterns of E74A RNA and protein expression at the onset of metamorphosis in Drosophila. Development 1991; 112: 981-995.

26. Daish TJ, Mills K, Kumar S. Drosophila caspase DRONC Is required for specific developmental cell death pathways and stress-induced apoptosis. Dev Cell 2004; 7: 909915.

27. Fernandez R, Tabarini D, Azpiazu N, Frasch M, Schlessinger J. The Drosophila insulin receptor homolog: a gene essential for embryonic development encodes two recepto isoforms with different signaling potential. EMBO J 1995; 14: 3373-3384

28. Whalen AM, Steward R. Dissociation of the dorsal-cactus complex and phosphorylation of the dorsal protein correlate with the nuclear localization of dorsal. J Cell Biol 1993; 123 : 523-534. 
29. Xue F, Cooley L. kelch encodes a component of intercellular bridges in Drosophila egg chambers. Cell 1993; 72: 681-693.

30. Tepass $U$, Theres $C$, Knust $E$. crumbs encodes an EGF-like protein expressed on apical membranes of Drosophila epithelial cells and required for organization of epithelia. Cell 1990; 61: 787-799.

31. Mahajan-Miklos S, Cooley L. The villin-like protein encoded by the Drosophila quail gene is required for actin bundle assembly during oogenesis. Cell 1994; 78 : 291-301.

32. Wan HI, DiAntonio A, Fetter RD, Bergstrom K, Strauss R, Goodman CS. Highwire regulates synaptic growth in Drosophila. Neuron 2000; 26: 313-329.

33. Collins RT, Furukawa T, Tanese N, Treisman JE. Osa associates with the Brahma chromatin remodeling complex and promotes the activation of some target genes. EMBO J 1999; 18: 7029-7040.

34. McCabe BD, Hom S, Aberle H, Fetter RD, Marques G, Haerry TE et al. Highwire regulates presynaptic BMP signaling essential for synaptic growth. Neuron 2004; 41: 891-905.
35. Chen J, Balgley BM, DeVoe DL, Lee CS. Capillary isoelectric focusing-based multidimensional concentration/separation platform for proteome analysis. Anal Chem 2003; 75: 3145-3152.

36. Lloyd TE, Atkinson R, Wu MN, Zhou Y, Pennetta G, Bellen HJ. Hrs regulates endosome membrane invagination and tyrosine kinase receptor signaling in Drosophila. Cell 2002; 108: 261-269.

37. Scott RC, Schuldiner O, Neufeld TP. Role and regulation of starvation-induced autophagy in the Drosophila fat body. Dev Cell 2004; 7: 167-178.

38. Justice RW, Zilian O, Woods DF, Noll M, Bryant PJ. The Drosophila tumor suppressor gene warts encodes a homolog of human myotonic dystrophy kinase and is required for the control of cell shape and proliferation. Genes Dev 1995; 9: 534-546.

39. Pantalacci S, Tapon N, Leopold P. The Salvador partner Hippo promotes apoptosis and cell-cycle exit in Drosophila. Nat Cell Biol 2003; 5: 921-927.

40. Akdemir F, Farkas R, Chen P, Juhasz G, Medved'ova L, Sass M et al. Autophagy occurs upstream or parallel to the apoptosome during histolytic cell death. Development 2006; 133: $1457-1465$.

Supplementary Information accompanies the paper on Cell Death and Differentiation website ( http://www.nature.com/cdd) 